\title{
Atrial fibrillation, sleep apnea and obesity
}

\author{
Mina K Chung*, Nancy Foldvary-Schaefer, Virend K Somers, Paul A Friedman and Paul J Wang
}

\section{SUMMARY}

Background A 60-year-old male with obesity (body-mass index $43 \mathrm{~kg} / \mathrm{m}^{2}$ ) presented with recurrent symptomatic atrial fibrillation (AF), which he had had since age 41 years. The AF was refractory to treatment with antiarrhythmic drugs. Pacemaker implantation for tachycardiabradycardia syndrome was required as well as ablation for atrial flutter, and the patient underwent a total of four DC cardioversions. Sleep studies showed mild to moderate obstructive sleep apnea, but continuous positive airway pressure was not tolerated. Pacemaker interrogations demonstrated mode-switch episodes, indicating continuing AF. He was scheduled for catheter ablation targeting pulmonary vein antral isolation. He embarked on a weight-loss program, which successfully reduced AF burden.

Investigations Echocardiography, stress testing, polysomnography, pacemaker interrogations and C-reactive protein.

Diagnosis AF, atrial flutter, tachycardia-bradycardia syndrome, obstructive sleep apnea and morbid obesity.

Management Antiarrhythmic drug therapy, DC cardioversion, anticoagulation, atrial flutter ablation, permanent pacemaker implantation, continuous positive airway pressure and weight loss.

KEYWORDS arrhythmia, atrial fibrillation, morbid obesity, obstructive sleep apnea, weight loss

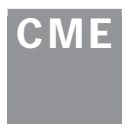

This article offers the opportunity to earn one Category 1 credit towards the AMA Physician's Recognition Award.

\section{THE CASE}

An obese man aged 41 years presented to his internist with episodic palpitations, dyspnea and fatigue. Paroxysmal AF was diagnosed and he was treated with digoxin, verapamil and quinidine. At age 51, AF recurred and persisted. Holter monitoring showed rates of $18-182$ beats/min and nocturnal pauses of up to $4.5 \mathrm{~s}$, consistent with tachycardia-bradycardia syndrome. Two DC cardioversion procedures, one with adjunctive quinidine, resulted in only transient sinus rhythm. Amiodarone was started and sinus rhythm was achieved, but recurrences of AF and type I atrial flutter required further DC cardioversion.

The patient was referred to a cardiac electrophysiologist for atrial flutter at age 56. Leftventricular, right-ventricular and right-atrial size and systolic function were normal on echocardiography; left-ventricular ejection fraction was $60 \%$ and mild left-ventricular hypertrophy and moderate left-atrial enlargement $(5.2 \mathrm{~cm})$ were present. A nuclear stress test showed no myocardial scar or ischemia. Another DC cardioversion and radiofrequency ablation of the right-atrial isthmus successfully treated atrial flutter.

Sinus rhythm was maintained 2 months later, and amiodarone was stopped. After another 3 months, symptomatic AF recurred that was refractory to flecainide, propafenone and dofetilide.

Because of a history of nocturnal bradycardia, morbid obesity and snoring, the patient was evaluated for OSA. He typically slept $7-8 \mathrm{~h}$ per night, experiencing three to four unexplained awakenings nightly. Sleep was unrefreshing and daytime sleepiness was moderate (EPWORTH SLEEPINESS SCALE score 15). At this time, body-mass index was $43 \mathrm{~kg} / \mathrm{m}^{2}$.

During overnight polysomnography, the patient slept for 196 of 361 min observed. No slow-wave sleep was recorded and there were 50 arousals, 23 APNEAS and eight HYPOPNEAS (Table 1). Mean 
oxygen saturation was 93\% (low of 76\%) and was $<90 \%$ for $3 \%$ of total sleep time. Loud snoring and snorting were reported. No notable arrhythmias were recorded. Continuous positive airway pressure (CPAP) $7 \mathrm{~cm} \mathrm{H}_{2} \mathrm{O}$ was attempted for 2 nights, but was discontinued due to discomfort.

Continuing rapid AF and bradycardia with nocturnal pauses limited drug therapy, and a dual-chamber pacemaker was implanted. AF became persistent 9 months later. Dofetilide was changed to sotalol with DC cardioversion, but 2 days later AF recurred and persisted. Pulmonary vein isolation was scheduled.

The patient lost weight to lessen complications in vascular access, imaging and catheter manipulation. After 1 month, AF spontaneously converted to sinus rhythm. By 3 months, he had lost $20.4 \mathrm{~kg}$; sinus rhythm was maintained at a weight of $125.6 \mathrm{~kg}$. The patient elected to cancel pulmonary vein isolation. Sotalol was reduced from $300 \mathrm{mg}$ to $240 \mathrm{mg}$ twice daily.

After 1 year, with further weight loss to $121.6 \mathrm{~kg}$ (body-mass index $37 \mathrm{~kg} / \mathrm{m}^{2}$ ), only two episodes of brief asymptomatic AF had been detected over the previous 8 months, and pulmonary vein isolation was indefinitely postponed. Table 2 displays the patient's clinical characteristics over time.

\section{DISCUSSION OF DIAGNOSES}

AF affects over 2 million people in the US, ${ }^{1}$ and prevalence is increasing. Apart from rapid palpitations, symptoms, including fatigue, dyspnea or weakness, can be subtle. Because of its association with thromboembolic complications, documentation of the arrhythmia is critical, even in asymptomatic AF. Electrocardiograms or ambulatory monitoring can detect arrhythmia. Holter and arrhythmia event monitors might also be useful. Echocardiography is indicated to assess for structural heart disease, which can increase the risk of stroke.

In lone $\mathrm{AF}$ with no structural heart disease and refractory to multiple antiarrhythmic drugs, the foci that initiate and possibly sustain the arrhythmia are generally located in the left atrium at the ostia of the pulmonary veins. ${ }^{2}$ AF frequently becomes more persistent over time, as in the case reported. Continued triggering of foci, electrical and structural remodeling leading to changes in ion-channel currents and atrial enlargement, changes in autonomic balance or myocardial senescence with accompanying structural and electrophysiological changes can perpetuate AF.

\begin{tabular}{lc}
$\begin{array}{l}\text { Table } 1 \text { Sleep disturbances recorded during } \\
\text { overnight polysomnography. }\end{array}$ \\
\hline Parameter of sleep disturbance & Score \\
\hline AROUSAL INDEX (arousals/sleep-hour) & 15 \\
\hline Apneas (number of events) & \\
\hline Central & 1 \\
\hline Mixed & 4 \\
\hline Obstructive & 18 \\
\hline Total & 23 \\
\hline Hypopneas (number of events) & 8 \\
\hline $\begin{array}{c}\text { Apnea-hypopnea index (apnea and } \\
\text { hypopnea events/sleep-hour) }\end{array}$ & \\
\hline Overall & 9.5 \\
\hline Supine & 18.6 \\
\hline
\end{tabular}

Atrial flutter is common in AF treated with antiarrhythmic drugs. Typical, or type I, atrial flutter is a macro re-entrant circuit in the right atrium with activation through the isthmus between the tricuspid annulus and inferior vena cava. It is characterized by 'saw-tooth' flutter waves in inferior electrocardiogram leads. Given the large excitable gap associated with typical atrial flutter, antiarrhythmic medications may stabilize re-entrant circuits. Atypical atrial flutter represents re-entrant circuits that are not dependent on the right atrial isthmus. The patient had classic tachycardia-bradycardia syndrome with periods of rapid rate during AF and slow rates during sinus rhythm, sleep or drug treatment.

OSA affects 10-15 million people in the US, and is diagnosed by sleep studies. ${ }^{3}$ Morbid obesity, history of snoring and nocturnal pauses possibly related to apnea, oxygen desaturation or hypervagal tone, led to OSA assessment in this patient. An association between AF and OSA has been studied. A case-control study reported no significant difference in the frequency of sleep apnea syndrome between lone AF patients and controls (32\% vs $29 \%),{ }^{4}$ although more daytime fatigue and nightly apnea occurred in the AF group. In a study of 151 patients undergoing DC cardioversion for AF compared with 312 patients without AF, however, OSA was diagnosed by questionnaire in more $\mathrm{AF}$ patients than in controls ( $49 \%$ vs $32 \%)^{5}$

OSA may impact the occurrence of AF by several mechanisms. Repetitive autonomic, hemodynamic and hypoxemic surges might activate stretch-mediated channels, catecholamine-

\section{GLOSSARY}

EPWORTH SLEEPINESS SCALE

A measure of daytime sleepiness based on a questionnaire in which responders rate the chance of dozing in eight activities

\section{APNEA}

A transient absence of spontaneous respiration

\section{HYPOPNEA}

Breathing that is shallower, slower or both, than normal

AROUSAL INDEX

The number of arousal events per sleep-hour 


\section{GLOSSARY APNEA-HYPOPNEA INDEX \\ The number of respiratory events (apneas and hypopneas) per sleep-hour}

Table 2 Clinical characteristics recorded from 15 December 2000 to 4 June 2004

\begin{tabular}{|c|c|c|c|c|c|c|}
\hline Date & $\begin{array}{l}\text { Weight } \\
\text { (kg) }\end{array}$ & $\begin{array}{c}\text { BMI } \\
\left(\mathrm{kg} / \mathrm{m}^{2}\right)\end{array}$ & Rhythm & $\begin{array}{l}\text { Number of } \\
\text { mode switches } \\
\text { ( } \% \text { mode } \\
\text { switched) }\end{array}$ & $\begin{array}{l}\text { CRP } \\
\text { (mg/l) }\end{array}$ & $\begin{array}{c}\text { Antiarrhythmic } \\
\text { drug }\end{array}$ \\
\hline 15 Dec 2000 & 137.7 & 42.3 & SR & N/A & 2.13 & Amiodarone \\
\hline 11 May 2001 & 145.5 & 44.6 & SR, PAF & N/A & 4.14 & None \\
\hline 20 July 2001 & 145.5 & 44.6 & AF & N/A & 2.80 & Flecainide \\
\hline 7 Sept 2001 & N/D & $\mathrm{N} / \mathrm{D}$ & SR, PAF & N/A & 3.21 & Dofetilide \\
\hline 5 Oct 2001 & 141.4 & 43.4 & SR, PAF & N/A & 3.57 & Dofetilide \\
\hline 16 Nov $2001^{a}$ & 138.2 & 42.4 & AF & 17 (29.9) & 3.04 & Dofetilide \\
\hline 21 May 2002 & 138.2 & 42.4 & SR, PAF & $84(15.8)$ & 2.79 & Dofetilide \\
\hline 8 July 2002 & N/D & N/D & AF & N/D & 3.20 & None \\
\hline 15 July 2002 & N/D & N/D & $\mathrm{AF}$ & N/D & 3.14 & Sotalol \\
\hline 18 Oct 2002 & 143.2 & 43.9 & AF & 137 (65.3) & 2.34 & Sotalol \\
\hline 24 Jan 2003 & 144.1 & 44.2 & AF & $15(69.7)$ & 1.90 & Sotalol \\
\hline 6 June $2003^{b c}$ & 146.4 & 44.9 & $\mathrm{AF}$ & $N / D$ & 2.70 & Sotalol \\
\hline 19 Sept $2003^{d}$ & 125.9 & 38.6 & SR & $N / D$ & 2.80 & Sotalol \\
\hline 4 June $2004^{e}$ & 121.8 & 37.4 & SR & $21(16)$ & 0.90 & Sotalol \\
\hline
\end{tabular}

sensitive channels or both, leading to greater focal discharges. Pulmonary or systemic hypertension, lung physiology restricted by morbid obesity or both can elevate atrial pressures and trigger ectopy and AF. Vagal stimulation of atrial myocardium shortens atrial refractory periods. Vagal reflexes during OSA, marked by bradyarrhythmias, could also reduce refractoriness at the pulmonary vein ostia, promoting conduction of focal discharges from the pulmonary vein to left atrium and generating a window for pulmonary vein foci to trigger AF.

Elevated C-reactive protein (CRP) has been associated with obesity, metabolic syndrome, severity of OSA and $\mathrm{AF}^{6}{ }^{6}$ The major determinant of CRP elevation in obese people may be the obesity, rather than OSA or metabolic syndrome. ${ }^{7}$ In the present case, initially high levels of CRP reduced with weight loss. Whether high concentrations associated with obesity indicate a predisposition to structural remodeling or AF itself provokes inflammation is unknown. Markers of inflammation reduce with weight loss of as little as $5 \%$ in obese subjects, ${ }^{8}$ and obesity, OSA or both could promote atrial structural remodeling via mechanical or inflammatory stress. The lag between body-mass index reduction and CRP and AF burden reduction observed in this patient suggests that more than 3 months might be required before improvements in mechanical, metabolic or inflammatory stressors are seen. Whether relief of OSA itself was the critical mechanical factor in this patient cannot be determined, as we did no follow-up sleep study after weight loss.

\section{TREATMENT AND MANAGEMENT}

AF management is aimed at reducing symptoms and the risk of thromboembolic complications. The Atrial Fibrillation Follow-up Investigation of Rhythm Management (AFFIRM) trial $^{9}$ demonstrated no survival benefits with a rhythmcontrol over rate-control strategy. The need for continued anticoagulation was confirmed in patients with risk factors for stroke, even with rhythm control and apparent maintenance of sinus rhythm. Warfarin anticoagulation was indicated for borderline hypertension and diabetes mellitus in the patient we describe.

Initial treatment comprised rate-controlling digoxin and verapamil and rhythm-controlling quinidine. First-line therapy has since changed to $\beta$-blockers; quinidine, which carries a risk of proarrhythmia, is usually avoided. When 
AF became more persistent, amiodarone was started. Although effective for AF, amiodarone can have toxic effects. Other first-line drugs for lone $\mathrm{AF}$ are flecainide and propafenone.

While taking amiodarone, the patient developed atrial flutter. Ablation of the posterior corridor that forms the circuit of atrial flutter may be used in conjunction with antiarrhythmic drugs. Sinus rhythm was maintained after ablation on amiodarone, but when amiodarone was stopped to avoid long-term toxic effects, AF recurred as expected. Flecainide, propafenone and dofetilide had no effect. DC cardioversions are helpful for persistent $\mathrm{AF}$ and can be performed multiple times.

Pacemaker implantation was chosen for symptomatic tachycardia-bradycardia syndrome to facilitate rate-controlling medications. Pacemakers may also be beneficial for OSA. ${ }^{10}$ Mode-switch logs enable assessment of AF burden.

Because of the persistence of the patient's AF, catheter-based pulmonary vein isolation was recommended. This method is successful in $60-85 \%$ of patients, with higher success rates in lone AF. Obesity can raise vascular access risks, such as inadvertent arterial cannulation or hematoma formation; doses of anticoagulation higher than normal might be required. In addition, weight limits of 135-160 kg often apply to catheterization tables, and fluoroscopy intensity can be raised. Weight loss is, therefore, advisable in obese patients scheduled for this procedure.

Mild OSA (APNEA-HYPOPNEA INDEX $<20$, without significant sleep complaints) can be managed conservatively with weight loss and avoidance of central nervous system depressants, sleep deprivation and sleeping supine. Even modest weight control has been shown to improve sleep-disordered breathing. ${ }^{11}$ CPAP should be offered as initial therapy in moderate to severe disease or mild disease with severe daytime sleepiness. Various interfaces should be tried for tolerability and comfort. Surgery should be considered if CPAP is not tolerated or fails.

When OSA and AF coexist, treatment for OSA might assist management of AF. Although a reduction in apneic episodes, bradyarrhythmias or both has been seen with atrial pacing or CPAP, supraventricular arrhythmias ${ }^{10,12}$ are not reduced. Nevertheless, a significantly raised risk of recurrent AF after DC cardioversion has been reported in untreated compared with treated patients with OSA. ${ }^{3}$ Risk of AF recurrence was particularly evident among untreated OSA patients with severe nocturnal oxygen desaturation, as in the patient we saw. Treatment or resolution of OSA could reverse the milieu that promotes AF and pulmonary vein to leftatrial connections. Whether pulmonary vein triggering foci become suppressed, latent or less prone to conduct to the left atrium with weight loss or OSA therapy requires further study.

\section{CONCLUSIONS}

In patients with $\mathrm{AF}$ and obesity, weight loss and screening for OSA should be pursued. Weight loss and treatment of OSA are likely to reduce mechanical, inflammatory and electrical stressors that promote AF. Equally importantly, screening for AF and appropriate treatment may help to reduce the risk of thromboembolic complications in OSA.

\section{References}

1 Fuster V et al. (2001) ACC/AHA/ESC Guidelines for the Management of Patients With Atrial Fibrillation: Executive Summary A Report of the American College of Cardiology/American Heart Association Task Force on Practice Guidelines and the European Society of Cardiology Committee for Practice Guidelines and Policy Conferences (Committee to Develop Guidelines for the Management of Patients With Atrial Fibrillation) Developed in Collaboration With the North American Society of Pacing and Electrophysiology. Circulation 104: 2118-2150

2 Haissaguerre M et al. (1998) Spontaneous initiation of atrial fibrillation by ectopic beats originating in the pulmonary veins. N Engl J Med 339: 659-666

3 Kanagala R et al. (2003) Obstructive sleep apnea and the recurrence of atrial fibrillation. Circulation 107: 2589-2594

4 Porthan KM et al. (2004) Prevalence of sleep apnea syndrome in lone atrial fibrillation: a case-control study. Chest 125: 879-885

5 Gami AS et al. (2004) Association of atrial fibrillation and obstructive sleep apnea. Circulation 110: 364-367

6 Chung MK et al. (2001) C-reactive protein elevation in patients with atrial arrhythmias: inflammatory mechanisms and persistence of atrial fibrillation. Circulation 104: 2886-2891

7 Aronson D et al. (2004) Obesity is the major determinant of elevated C-reactive protein in subjects with the metabolic syndrome. Int $\mathrm{J}$ Obes Relat Metab Disord 28: 674-679

8 Valsamakis G et al. (2004) Modest weight loss and reduction in waist circumference after medical treatment are associated with favorable changes in serum adipocytokines. Metabolism 53: 430-434

9 Wyse DG et al. (2002) A comparison of rate control and rhythm control in patients with atrial fibrillation. N Engl J Med 347: 1825-1833

10 Garrigue S et al. (2002) Benefit of atrial pacing in sleep apnea syndrome. N Engl J Med 346: 404-412

11 Peppard PE et al. (2000) Longitudinal study of moderate weight change and sleep-disordered breathing. JAMA 284: 3015-3021

12 Simantirakis EN et al. (2004) Severe bradyarrhythmias in patients with sleep apnoea: the effect of continuous positive airway pressure treatment; A long-term evaluation using an insertable loop recorder. Eur Heart $J$ 25: 1070-1076

\section{Acknowledgments} Written consent for publication was obtained from the patient reported in this case study.

Competing interests The authors declared they have no competing interests. 